\title{
Battery-Powered Digital CMOS Design
}

\author{
Massoud Pedram, Qing Wu \\ Department of Electrical Engineering-Systems \\ University of Southern California, Los Angeles, California, USA \\ \{pedram,qwu\}@usc.edu
}

\begin{abstract}
In this paper we study tradeoffs between energy dissipation and delay in battery-powered digital CMOS designs. In contrast to previous work, we adopt an integrated model of the VLSI circuit and the battery sub-system that powers it. We show that accounting for the dependence of battery capacity on the average discharge current changes shape of the energy-delay trade-off curve and hence the value of the operating voltage that results in the optimum energy-delay product for the target circuit. Analytical derivations as well as experimental results demonstrate the importance of correct modeling of the battery-hardware system as a whole and provide a more accurate basis for comparing various low power optimization methodologies and techniques targeted toward battery-powered electronics.
\end{abstract}

\section{INTRODUCTION}

Due to rapid progress in the semiconductor process technology, the device density and operating frequency have greatly increased, making power consumption in digital circuits a major design concern. High power consumption reduces the battery life in portable devices. The goal of low-power design for batteryoperated devices is to extend the battery lifetime while meeting the required performance specification.

The most effective method for low-power design is to reduce the supply voltage and compensate for the performance loss by a combination of architectural and circuit optimization techniques. Static voltage scaling [1][2] and dynamic voltage scaling [3] techniques have been proposed. It is important to evaluate the proposed techniques by using appropriate metrics, i.e., power, energy, delay, or energy-delay product. These metrics can be used in different applications (depending on the design requirements) to guide optimizations toward the best solution. It has been argued in [2] that the energy-delay product is more relevant for the purpose of comparing various low power design methodologies and techniques.

Battery Sub-system

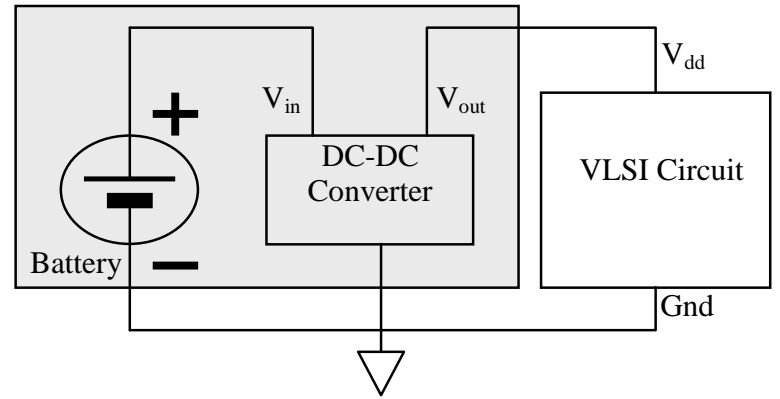

Figure 1 A complete battery operated system.

As shown in Figure 1, a battery-powered digital system (which is typically present in portable electronic devices such as cellular phones, notebook computers, PDA's) consists of the VLSI circuit, the battery cell, and the DC-DC converter. Although low-power design for portable electronics aims at extending the battery life, discussions of low-power-design metrics have entirely focused on the VLSI circuit itself, assuming that the battery system is an ideal source that outputs a constant voltage and stores/delivers a fixed amount of energy [4]. However, in reality, the energy stored in a new primary (non-rechargeable) battery or a fully charged secondary (rechargeable) battery cannot be extracted/used to the full extent. In fact, in some cases, even $50 \%$ energy delivery is not possible. This phenomenon is caused by the fact that the "actual capacity" of the battery depends strongly on the mean value of the current discharged from the battery. More precisely, a higher portion of the total battery capacity is wasted at higher discharge current. High rate (current) discharge can indeed cause dramatic (more than 50\%) waste of the initial capacity (energy) of the battery [6]. Notice that some energy is also wasted in the DC/DC converter. This is however relatively small and independent of the output current demand for a well-designed DC/DC converter [5].

In this paper, we adopt the energy-delay metric to evaluate low power digital designs. However, we depart from [2] by considering a first-order model of the battery sub-system which powers the VLSI circuit and show that the basic energy-delay tradeoff curve will change as a result of this integrated battery-hardware model. We thereby provide better insight into some of the basic tradeoffs that exist in battery-operated low power digital designs. We therefore show that, for battery-operated circuits, discussion of power-speed trade-off will be incomplete and inaccurate if we only consider the characteristics of the VLSI circuit.

The paper is organized as follows. Section II introduces some background knowledge. Section III gives the analytical form of the energy-delay product using an integrated battery-hardware model. Section IV presents the experimental results and discussions. Section V discusses the problem of optimal battery selection for a given VLSI circuit. Section VI gives our conclusions.

\section{BACKGROUND}

Different types of batteries are being used in a wide range of applications [6]-[14]. They can be classified into the primary batteries (non-rechargeable) and the secondary batteries (rechargeable). Batteries can also be classified based on the electrochemical material used for their electrodes or the type of their electrolytes, e.g., Ni-Cd, Ni-Zn, Ag-Zn, Zn-Air, NiMH, Lithium-Ion, Lithium Alloy Polymer, etc.. Among these, the NiMH battery and the Lithium-Ion battery are currently the most popular batteries for portable computers.

Figure 2 taken from [6] shows the internal structure of a typical rechargeable lithium battery. It consists of a lithium foil anode, a composite cathode, and an electrolyte that serves as an ionic path between electrodes and separates the two materials. Electronic energy is generated by chemical reaction among these three components. For rechargeable batteries, applying electrical 
recharging can reverse chemical reaction, hence the battery can be used for multiple times (normally several hundred times).

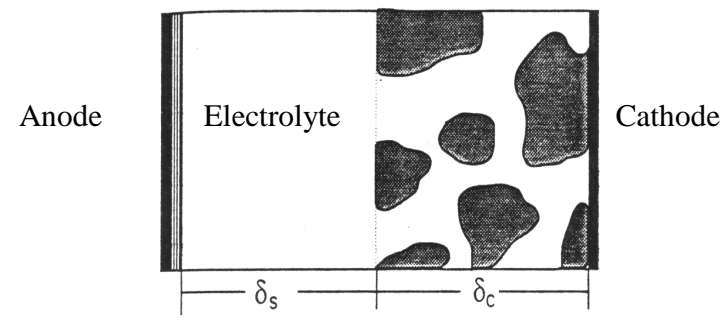

Figure 2 The internal structure of a battery.

Figure 3 [5] shows the block diagram of a high-efficiency DC-DC converter that can be integrated on a chip. Node BVdd is the input of the DC-DC converter which is connected to the positive electrode of the battery. Node CVdd is the output of the DC-DC converter which is connected to the VLSI circuit. The circuit level diagram for the Buck Converter is also shown. Other components are used for adaptively generating the switching signals for the Buck Converter such that the voltage at CVdd is stabilized at the target supply voltage for the VLSI circuit.

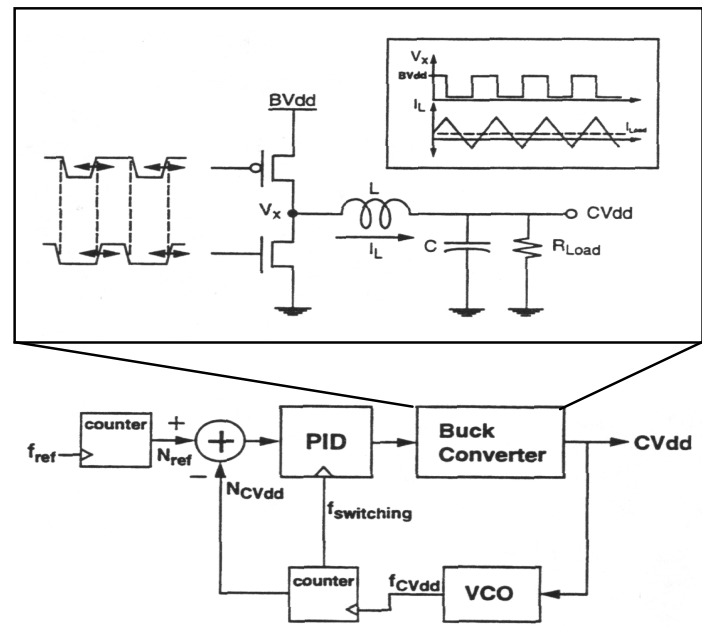

Figure 3 The structure of a DC-DC converter.

\section{A. Low Power Design Metrics}

The delay of a CMOS circuit can be estimated as [1]:

$$
t_{d}=k \frac{C V_{d d}}{\left(V_{d d}-V_{t h}\right)^{2}}
$$

where $k$ is some positive constant, $C$ is the loading capacitance, $V_{d d}$ is the supply voltage of the circuit, and $V_{t h}$ is the magnitude of the threshold voltage of a CMOS transistor.

The energy needed to complete a fixed-latency operation (e.g.. a $0 \rightarrow 1$ transition) is calculated as [1]:

$$
E_{o p}=n C V_{d d}^{2}
$$

where $n$ is some positive constant, $C$ is the loading capacitance, and $V_{d d}$ is the supply voltage.

The energy-delay product (EDP) metric is then:

$$
E D P_{o p}=k^{\prime \prime} \cdot \frac{V_{d d}^{3}}{\left(V_{d d}-V_{t h}\right)^{2}}
$$

where $k^{\prime \prime}=k \cdot n \cdot C$.

Notice that Equations (2.1), (2.2) and (2.3) are general enough such that they can used for representing $t_{d}$ and $E_{o p}$ for the whole circuit and for complex operations as well as for one single gate and one single transition. Figure 4 shows energy, delay, and energy-delay product curves versus the supply voltage. The minimum value of the energy-delay product occurs at $V_{d d}=3 V_{t h}[1]$.

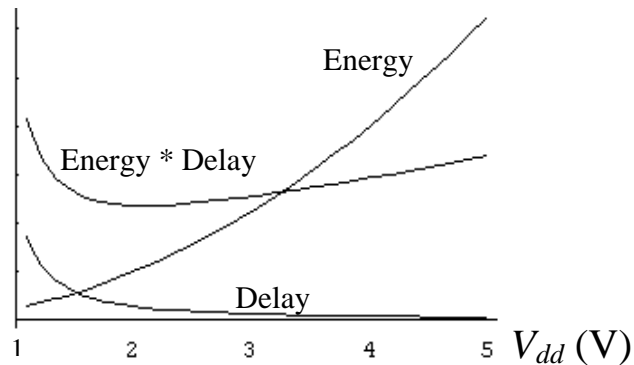

Figure 4 Energy and Delay vs. Supply Voltage $\left(V_{t h}=0.7 \mathrm{~V}\right)$.

\section{B. Battery Capacity}

We define $C A P_{0}$ as the amount of energy that is stored in a new primary battery or a fully charged secondary battery. The actual capacity $C A P_{a c t}$ is defined as:

$$
C A P_{a c t}=C A P_{0} \cdot \mu, \quad 0<\mu<1
$$

where $\mu$ is called the efficiency (or utilization) factor. $C A P_{a c t}$ is the actual energy that can be output by the battery.

The efficiency factor $\mu$ is a function of discharge current $I$ :

$$
\mu=f(I)
$$

where $f$ is a monotonic-decreasing function [6]. Only the lowfrequency part of the current is relevant to changing the battery efficiency [14]. Therefore, $I$ represents the time-averaged output current of the battery. The actual capacity of the battery will decrease when the discharge current increases.

Figure 5 shows the efficiency factor versus discharge current curve for some commercial NiMH batteries [12]. Similar curves exist for lithium batteries [13]. Notice that these curves are obtained for a constant current discharge. Batteries have a low pass filter at their output, which filters out fast transients on the circuit current, thereby, keeping the battery discharge current relatively fixed over some period of time (which is in order of $m s$ ).

To obtain an analytical form, two approximation functions will be used in this paper: linear and quadratic. With the linear approximation, Eqn. (2.5) is written as:

$$
\mu=1-\alpha \cdot I
$$

where $\alpha$ is a positive constant number.

With the quadratic approximation, Eqn. (2.5) is written as:

$$
\mu=1-\alpha \cdot I^{2}
$$




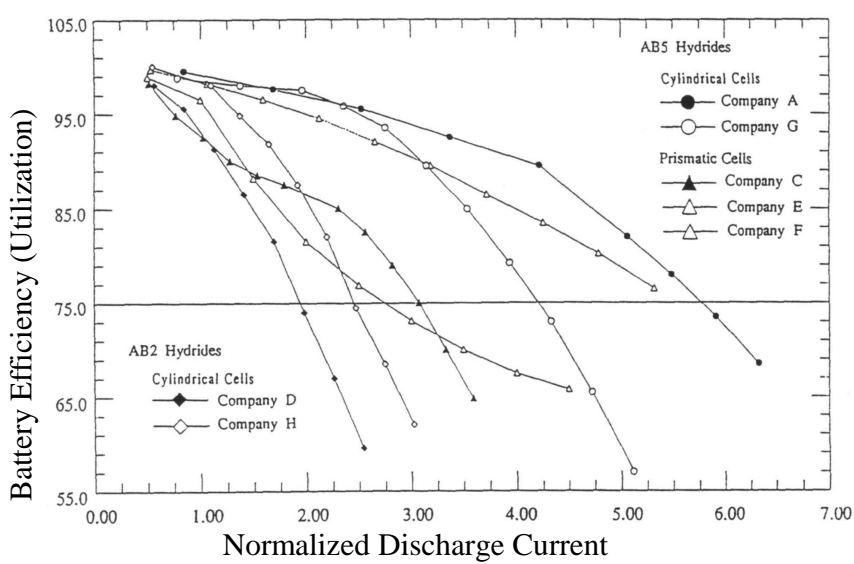

Figure 5 Efficiency factor versus discharge current.

\section{ENERGY-DELAY PRODUCT}

We first give some useful notation:

$T$ : Computation time for one operation

$V_{0}$ : Output voltage of the battery

$I_{0}$ : Output current of the battery

$\overline{I_{0}}$ : Average battery current over time $T$

$V_{d d}$ : Supply voltage of the circuit

$I_{d d}$ : Supply current of the circuit

$\overline{I_{d d}}:$ Average circuit current over time $T$

$\mu$ : Efficiency factor of the battery

$\eta$ : Efficiency of the DC-DC converter

$E_{o p}$ : The ideal energy needed for one operation

$E_{o p}^{a c t}:$ The actual (battery) energy needed for one operation

Notice that $V_{0}, V_{d d}$ and $\eta$ remain constant during $T$. An operation may refer to a single binary transition or a complex RT-level operation.

\section{A. The Effective Energy per Operation}

From Eqn. (2.2), the energy per operation consumed by the circuit can be written as:

$$
\begin{aligned}
& E_{o p}=n C V_{d d}^{2}=\int_{0}^{T} V_{d d} \cdot I_{d d} \cdot d t \\
& =V_{d d} \cdot \int_{0}^{T} I_{d d} d t=V_{d d} \cdot \overline{I_{d d}} \cdot T
\end{aligned}
$$

Notice that actual $I_{d d}$ is a function of time.

We can write the following equation for the DC-DC conversion:

$$
\eta \cdot V_{0} \cdot I_{0}=V_{d d} \cdot I_{d d} \quad \text { or } \eta \cdot V_{0} \cdot \overline{I_{0}}=V_{d d} \cdot \overline{I_{d d}}
$$

The actual energy per operation is calculated as:

$$
E_{o p}^{a c t}=\int_{0}^{T} \frac{V_{0} \cdot I_{0}}{\mu} d t
$$

Because $\mu$ is a function of the average (and not the instantaneous) value of $I_{0}$, we can write Eqn. (3.3) as:

$$
E_{o p}^{a c t}=\frac{V_{0} \cdot \overline{I_{0}} \cdot T}{f\left(\overline{I_{0}}\right)}
$$

Substituting equations (3.1) and (3.2), we obtain:

$$
E_{o p}^{a c t}=\frac{V_{0} \cdot T \cdot \frac{E_{o p} / T}{V_{0} \cdot \eta}}{f\left(\frac{E_{o p} / T}{V_{0} \cdot \eta}\right)}=\frac{1}{\eta} \cdot \frac{n C V_{d d}^{2}}{f\left(\frac{n C V_{d d}^{2}}{V_{0} \cdot \eta \cdot T}\right)}
$$

If we replace $f$ in (3.5) by either (2.6) or (2.7), we can write the actual energy per operation as a function of the supply voltage $V_{d d}$. If we use Eqn. (2.6), Eqn. (3.5) becomes:

$$
E_{o p}^{a c t}=\frac{1}{\eta} \cdot \frac{n C V_{d d}^{2}}{1-\alpha \cdot \frac{n C V_{d d}^{2}}{V_{0} \cdot \eta \cdot T}}
$$

If we use Eqn. (2.7), we get:

$$
E_{o p}^{a c t}=\frac{1}{\eta} \cdot \frac{n C V_{d d}^{2}}{1-\alpha \cdot\left(\frac{n C V_{d d}^{2}}{V_{0} \cdot \eta \cdot T}\right)^{2}}
$$

From (3.6) and (3.7), we know that the actual energy dissipation $E_{o p}^{a c t}$ is always larger than the ideal energy dissipation $E_{o p}$. The larger the $V_{d d}$ is, the larger is the difference. Figure 6 shows the comparison of $E_{o p}$ and $E_{o p}^{a c t}$ as a function of $V_{d d}$.

Due to space limitation, we use Eqn. (3.6) for the rest of the analysis in the section. Analysis using Eqn. (3.7) is similar.

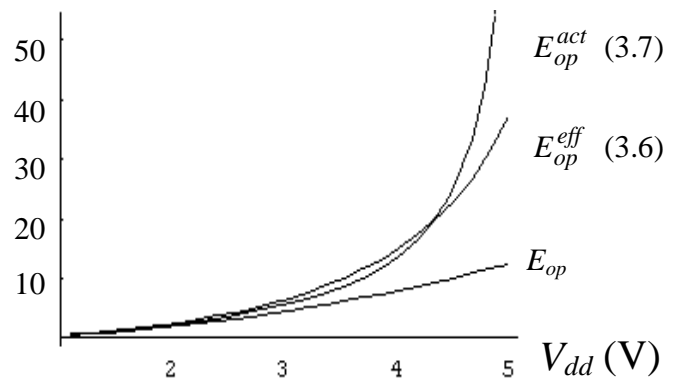

Figure 6 Comparison of $E_{o p}$ and $E_{o p}^{a c t}$.

\section{B. The Energy-Delay-Product Metric}

The energy-delay product (in brief, EDP) can be written as:

$$
E D P_{o p}^{a c t}=k \cdot \frac{V_{d d}}{\left(V_{d d}-V_{t h}\right)^{2}} \cdot \frac{1}{\eta} \cdot \frac{n C V_{d d}^{2}}{1-\alpha \cdot \frac{n C V_{d d}^{2}}{V_{0} \cdot \eta \cdot T}}
$$




$$
E D P_{o p}^{a c t}=k^{\prime} \cdot \frac{V_{d d}^{3}}{\left(V_{d d}-V_{t h}\right)^{2}\left(1-\beta V_{d d}^{2}\right)}
$$

where $k^{\prime}=\frac{k \cdot n \cdot C}{\eta}$ and $\beta=\frac{\alpha \cdot n \cdot C}{V_{0} \cdot \eta \cdot T}$

To give a quantitative comparison between $E D P_{o p}$ and $E D P_{o p}^{a c t}$, we assign reasonable values to the parameters as follows: $\eta$, the efficiency of the DC-DC converter, is taken to be 90\% [5], $V_{t h}=0.7 \mathrm{~V}$ and $V_{0}=4 \mathrm{~V}$. Assuming a circuit with $V_{d d}=3.3 \mathrm{~V}$ and average power dissipation of $33 \mathrm{~W}$, we obtain $(n C / T)=3$. $\alpha=0.05$ is a reasonable value for the battery $(50 \%$ efficiency at a $10 \mathrm{~A}$ discharge current) [12]. Therefore, a reasonable value for $\beta$ is around 0.04 (notice that the value for $\beta$ must satisfy: $0<1-\beta V_{d d}^{2}<1$ ). Since the absolute value of $k$ ' and $k$ " does not influence the solution of $V_{d d}$ for minimum EDP, we set $k^{\prime \prime}=1$, therefore $k^{\prime}=1 / 0.9$. Figure 7 shows the plots of $E D P_{o p}$ and $E D P_{o p}^{e f f}$ for different $\beta$ values. Table 1 shows the solution for $V_{d d}$ values that minimize the EDP.

The analytical derivation indicates that after combining the battery system with the VLSI circuit, the optimal $V_{d d}$ for minimum energydelay product becomes smaller than the ideal case that does not consider the battery system. The optimal $V_{d d}$ may change depending on the discharge characteristics of the battery. A larger $\beta$ implies a larger value of $\alpha$, which means the battery efficiency decreases faster when the current increases. Therefore, we conclude that when $\alpha$ increases, the value of optimal $V_{d d}$ becomes smaller. For the typical $\beta$ value of 0.04 , the optimal $V_{d d}$ is $17 \%$ smaller than the optimal $V_{d d}$ for the ideal case.

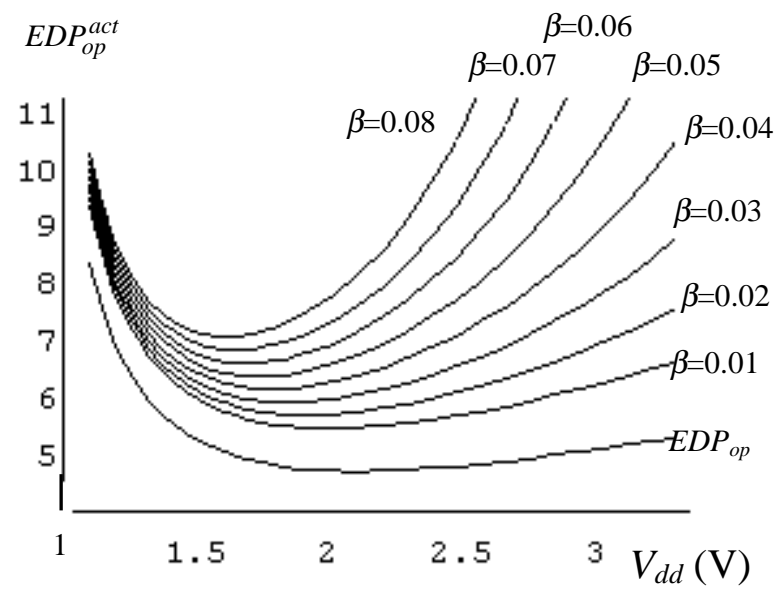

Figure 7 Plots of $E D P_{o p}$ and $E D P_{o p}^{a c t}$ with different $\beta$ values.

Table 1 Solution of $V_{d d}$ for minimum EDP.

\begin{tabular}{|c|c|c|c|c|c|c|c|c|c|}
\hline & $E^{2} P_{o p}$ & \multicolumn{10}{|c|}{$E D P_{o p}^{a c t}$} \\
\hline$\beta$ & & 0.01 & 0.02 & 0.03 & 0.04 & 0.05 & 0.06 & 0.07 & 0.08 \\
\hline$V_{d d} *(\mathrm{~V})$ & 2.1 & 2.0 & 1.9 & 1.8 & 1.75 & 1.71 & 1.68 & 1.66 & 1.65 \\
\hline
\end{tabular}

IV. EXPERIMENTAL RESULTS

For the experimental setup, we designed a small system where the VLSI circuit is represented by an optimally sized 4-inverter buffer with a capacitive load of $0.5 \mathrm{pF}$. A $0.5 \mu$ CMOS process technology was used for the transistor models. A macro-model of the battery was generated following the model proposed by [14]. The battery capacity was scaled down to reduce the simulation time, as well as to match the scaled-down size of the VLSI circuit. An appropriate macro-model was used for the DC-DC converter simulation. The efficiency of the converter was set to $90 \%$ for converting $V_{0}$ to different $V_{d d}$ 's. We used HSPICE to generate the experimental results.

\section{A. Ideal Battery Model}

To obtain the various curves for the ideal case, we simulated the circuit for one clock cycle with an ideal voltage source with different $V_{d d}$ values. Energy and delay values were measured during the simulation, energy-delay product values were subsequently calculated from these. The plots of these metrics versus $V_{d d}$ are shown in Figure 8.

Experimental results show that the $V_{d d}$ for minimum energy-delay product is about $2.0 \mathrm{~V}$, which is close to the analytical result $(2.1 \mathrm{~V})$.

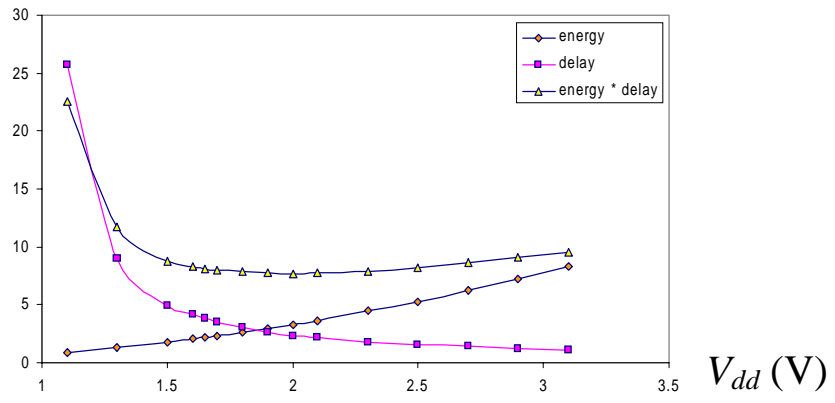

Figure 8 Energy (pJ), delay (ns), energy-delay (pJ*ns) plots for the ideal battery model (same scale).

B. Real Battery Model

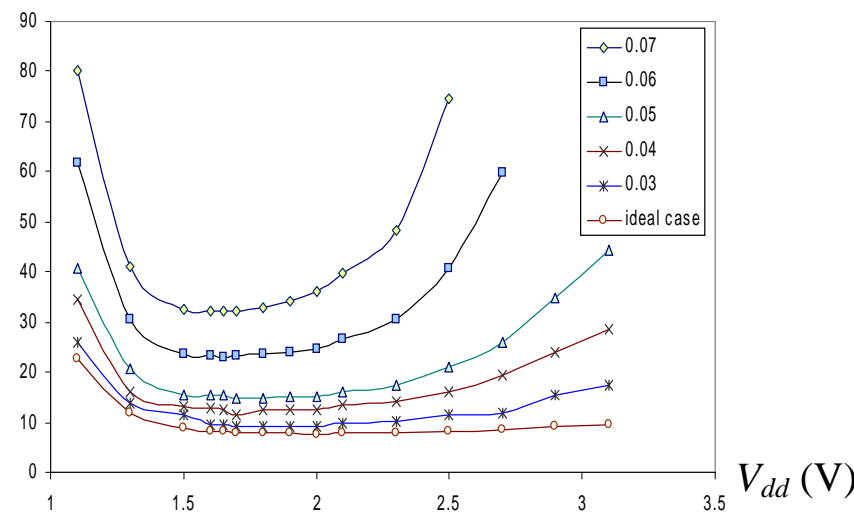

Figure 9 Plots of $E D P_{o p}$ and $E D P_{o p}^{a c t}(\mathbf{p J} * \mathbf{n s})$.

In this setup, we want to measure the actual energy per operation $E_{o p}^{a c t}$ for the system. Then we can use the delay measurement from the previous sub-section to obtain the plot of the actual energydelay product $E D P_{o p}^{a c t}$. Since we could not measure an abstract quantity directly, we use the following relation:

$$
\mathrm{E}_{\mathrm{op}}^{\mathrm{act}}=\mathrm{CAP}_{0} / \mathrm{N}
$$


where $N$ represents the number of operations that the circuit performs before the battery is depleted. It can easily be measured by simulation. Batteries with different $\alpha$ values are simulated to make similar plots as in Figure 7 and Table 1. The results are reported in Figure 9 and Table 2.

Table 2 Optimal $V_{d d}$ from experimental results.

\begin{tabular}{|c|c|c|c|c|c|c|}
\hline & $E D P_{o p}$ & \multicolumn{5}{|c|}{$E D P_{o p}^{a c t}$} \\
\hline$\beta$ & & 0.03 & 0.04 & 0.05 & 0.06 & 0.07 \\
\hline$V_{d d} *(\mathrm{~V})$ & 2.0 & 1.9 & 1.75 & 1.7 & 1.65 & 1.6 \\
\hline & V. BATTERY SELECTION
\end{tabular}

We showed in the previous section that the battery characteristics change the optimal value of $V_{d d}$ for a VLSI circuit. Similarly, the characteristics of the VLSI circuit can influence choice of the battery for the circuit. The goal of battery selection process is to find the battery that can make the given system work longest within one battery cycle (time from new or fully charged battery to battery replacement or recharge). If we define the battery life as the number of operations the system can perform before the battery is totally discharged, our goal is to find the battery which maximizes $N$ as defined in Section IV. Of course there are other considerations for battery selection such as weight and size. We assume that those constraints have also been considered for the selection of the appropriate battery and that we have a tie with respect to those criteria.

Under the ideal battery model, we have:

$$
\mathrm{N}=\mathrm{CAP}_{0} / \mathrm{E}_{\text {op }}
$$

Since $E_{o p}$ is known (and fixed) for the given circuit, to maximize $N$, we must simply maximize $C A P_{0}$. Therefore, the criterion for battery selection is very simple: select the battery with maximum capacity $C A P_{0}$.

Under the real battery model, we have:

$$
\mathrm{N}=\mathrm{CAP}_{0} / \mathrm{E}_{\mathrm{op}}^{\mathrm{act}}
$$

Substituting Eqn. (3.6) into (5.2) and noting that $\eta, V_{d d}, I_{d d}$, and $T$ have been determined by the design of the DC-DC converter and the VLSI circuit, we can rewrite (5.2) as:

$$
N=\frac{C A P_{0} \cdot\left(1-\frac{\alpha}{V_{0}} \cdot k\right)}{k \cdot T}
$$

where $k=\frac{V_{d d} \cdot \overline{I_{d d}}}{\eta}$ is the amount of battery output power required by the DC-DC converter.

From Eqn. (5.4), we can see that to maximize $N$, we need to maximize $C A P_{0} \cdot\left(1-\frac{\alpha}{V_{0}} \cdot k\right)$. Recall that $\alpha$ and $V_{0}$ are important performance parameters for batteries. Therefore, a battery with the largest $C A P_{0}$ may not be the best choice. As an example, if all candidate batteries have the same $C A P_{0}$, we should choose the one with smallest value of $\alpha / V_{0}$.

\section{CONCLUSION}

In this paper, we showed that it is essential to consider the characteristics of the battery that powers a portable electronic circuit in deciding the effectiveness of various low power optimization techniques. We also proposed a simple, yet accurate, integrated model of the battery and VLSI sub-systems. Next we studied (analytically and empirically) the problem of assigning a voltage level to the VLSI circuit which minimizes the effective (actual) energy-delay product in the combined system. Finally we considered the problem of battery selection for given VLSI circuit (with fixed supply voltage level and energy per operation cost). Next step is to consider the battery-hardware co-design problem for battery-powered electronic systems.

\section{ACKNOWLEDGEMENT}

We would like to thank Qinru Qiu for helpful discussions.

\section{REFERENCES}

[1] A. Chandrakasan, R. Brodersen, Low Power Digital CMOS Design, Kluwer Academic Publishers, July 1995.

[2] M. Horowitz, T. Indermaur, and R. Gonzalez, "Low-Power Digital Design”, IEEE Symposium on Low Power Electronics, pp.8-11, 1994.

[3] A. Chandrakasan, V. Gutnik, and T. Xanthopoulos, "Data Driven Signal Processing: An Approach for Energy Efficient Computing", 1996 International Symposium on Low Power Electronics and Design”, pp. 347-352, Aug. 1996.

[4] J. Rabaey and M. Pedram, Low Power Design Methodologies, Kluwer Academic Publishers, 1996

[5] G. Wei and M. Horowitz, "A Low Power Switching Power Supply for Self-Clocked Systems", 1996 International Symposium on Low Power Electronics and Design", pp. 313-317, Aug. 1996.

[6] M. Doyle, T. F. Fuller, and J. Newman, "Modeling of Galvanostatic Charge and Discharge of the Lithium/Polymer/Insertion Cell", $J$. Electrochem. Soc., Vol. 140, No. 6, pp.1526-1533, Jun. 1993.

[7] T. F. Fuller, M. Doyle, and J. Newman, "Simulation and Optimization of the Dual Lithium Ion Insertion Cell", J. Electrochem. Soc., Vol. 141, No. 1, pp.1-9, Jan. 1994.

[8] D. Fauteux, "Lithium Polymer Electrolyte Rechargeable Battery", The Electrochemical Society Proceedings, Vol. 94-28, pp.379-388.

[9] L. Xie, W. Ebner, D. Fouchard, and S. Megahed, "Electrochemical Studies of $\mathrm{LiNiO}_{2}$ for Lithium-Ion Batteries", The Electrochemical Society Proceedings, Vol. 94-28, pp.263-276.

[10] K. M. Abraham, D. M. Pasquariello, T. H. Nguyen, Z. Jiang, and D. Peramunage, "Lithiated Manganese Oxide Cathodes for Rechargeable Lithium Batteries", The Battery Conference, pp. 317-323, 1996.

[11] N. Cui, B. Luan, D. Bradhurst, H. K. Liu, and S. X. Dou, "SurfaceModified $\mathrm{Mg}_{2} \mathrm{Ni}$-Type Negative Electrode Materials for $\mathrm{Ni}-\mathrm{MH}$ Battery", The Battery Conference, pp. 317-322, 1997.

[12] J. K. Erbacher and S. P. Vukson, "Commercial Nickel-Metal Hydride (Ni-MH) Technology Evaluation", The Battery Conference, pp. 9-15, 1997

[13] B. Nelson, "TMP Ultra-High Rate Discharge Performance", The Battery Conference, pp. 139-143, 1997.

[14] S. Gold, "A PSPICE Macromodel for Lithium-Ion Batteries", The Battery Conference, pp. 215-222, 1997

[15] URL: http://www.valence-tech.com/products/index.htm. 